\title{
microRNA expression signature of gastric cancer cells relative to normal gastric mucosa
}

\author{
BEI-QIN YU, LI-PING SU, JIAN-FANG LI, QU CAI, MIN YAN, XUE-HUA CHEN, YING-YAN YU, \\ QIN-LONG GU, ZHENG-GANG ZHU and BING-YA LIU
}

\begin{abstract}
Shanghai Key Laboratory of Gastric Neoplasms, Department of Surgery, Shanghai Institute of Digestive Surgery, Ruijin Hospital, Shanghai Jiao Tong University School of Medicine, Shanghai 200025, P.R. China
\end{abstract}

Received January 16, 2012; Accepted July 20, 2012

DOI: $10.3892 / \mathrm{mmr} .2012 .1006$

\begin{abstract}
RNAs (miRNAs) play an important role in a wide range of physiological and developmental processes by negatively regulating the expression of target genes at the post-transcriptional level. In this study, we investigated the differential miRNA expression signature between gastric cancer cells and normal gastric mucosa to determine changes in miRNA expression during gastric cancer development. We analyzed the global miRNA expression profiles of 9 gastric cancer cell lines and 6 normal gastric mucosa lines using miRNA microarrays. In addition, we performed quantitative real-time PCR (Q-PCR) to validate the results. Correlations between the miRNA expression profile and tumor clinicopathological parameters were analyzed. We found that 17 miRNAs were upregulated in gastric cancer cell lines and 146 miRNAs were downregulated compared to normal gastric mucosa. Using microarray data and Q-PCR validation, 15 miRNAs were finally selected. These candidate miRNAs were associated with gastric cancer clinicopathology to various degrees. High expression levels of hsa-miR-93 were found to predict poor survival (median, 16 vs. 40 months; log-rank test $\mathrm{p}<0.05$ ). These findings suggest that miRNAs play vital roles in human gastric cancer. The findings may also provide clues toward understanding the molecular functions of miRNAs in various biological processes.
\end{abstract}

\section{Introduction}

Gastric cancer, the second most common cancer in the world, is responsible for $12 \%$ of all cancer-related mortality

Correspondence to: Professor Bing-Ya Liu, Shanghai Key Laboratory of Gastric Neoplasms, Department of Surgery, Shanghai Institute of Digestive Surgery, Ruijin Hospital, Shanghai Jiao Tong University School of Medicine, No. 197 Ruijin Er Road, Shanghai 200025, P.R. China

E-mail: liubyrj@yahoo.com.cn

Key words: microRNAs, gastric cancer, microarray, quantitative real-time PCR each year. Therefore, improved treatments based on a deeper understanding of the molecular mechanisms underlying the progression of this disease are required (1). The pathogenesis and mechanisms behind gastric cancer development remain unknown. microRNAs (miRNAs) are small non-coding RNAs that regulate the expression of approximately $30 \%$ of all human genes by inhibiting target mRNA translation and inducing mRNA degradation $(2,3)$. miRNAs are capable of regulating a wide range of physiological and developmental processes, including cancer initiation and progression (4,5). Genome-wide studies have demonstrated that miRNA genes are frequently located at cancer-associated genomic regions or fragile sites, in minimal regions of loss of heterozygosity, regions of amplification and in common breakpoint regions, indicating their potential roles in tumorigenesis (6). Aberrant miRNA expression has also been frequently reported in various tumors such as B cell chronic lymphocytic leukemia, lung, breast and colon cancer, hepatoma and glioblastoma (7-10), indicating a close correlation between miRNA and cancer $(11,12)$. Taken together, these previous studies indicate that miRNAs are rich in information with respect to cancer and that a large amount of diagnostic information will be available once the relative concentrations of the involved miRNAs become known. The miRNA expression signatures are associated with well-defined clinicopathological features in human cancers.

Microarray experiments may reveal both increases and decreases in the production of various miRNAs. The expression pattern appears to be tissue-specific; different types of tumor have distinctive patterns of miRNA expression (13). Determination of the miRNA expression profile in cancer may lead to a better understanding of the genetic pathways involved in tumor development.

\section{Materials and methods}

Cell lines. Gastric cancer cell lines NCI-N87 (ATCC: CRL-5822), SNU-1 (ATCC: CRL-5971), SNU-16 (ATCC: CRL-5974), AGS (ATCC: CRL-1739) and KATOIII (ATCC: HTB-103) were obtained from the American Type Culture Collection (Manassas, VA, USA). Another 4 gastric cancer cell lines, MKN45, MKN28, BGC-823 and SGC-7901, were preserved at our institute. All cell lines were maintained in RPMI-1640 supplemented with 10\% fetal bovine serum (FBS). 
Table I. Relative expression of 46 miRNAs in gastric cancer cell lines and normal gastric mucosa.

\begin{tabular}{|c|c|c|c|c|}
\hline \multirow[b]{2}{*}{ No. } & \multicolumn{2}{|c|}{ Upregulated miRNAs } & \multicolumn{2}{|c|}{ Downregulated miRNAs } \\
\hline & miRNA & Log ratio & miRNA & Log ratio \\
\hline 1 & hsa-miR-196a & 5.69 & hsa-miR-376a & -12.52 \\
\hline 2 & hsa-miR-615 & 4.24 & hsa-miR-145 & -12.49 \\
\hline 3 & hsa-miR-196b & 4.20 & hsa-miR-143 & -12.44 \\
\hline 4 & hsa-miR-18a* & 2.84 & hsa-miR-451 & -10.38 \\
\hline 5 & hsa-miR-92b & 2.59 & hsa-miR-142-5p & -9.62 \\
\hline 6 & hsa-miR-149 & 2.38 & hsa-miR-1 & -8.94 \\
\hline 7 & hsa-miR-550 & 2.37 & hsa-miR-377 & -8.85 \\
\hline 8 & hsa-miR-183 & 1.95 & hsa-miR-495 & -8.79 \\
\hline 9 & hsa-miR-301 & 1.88 & hsa-miR-409-3p & -8.71 \\
\hline 10 & hsa-miR-18a & 1.81 & hsa-miR-368 & -8.54 \\
\hline 11 & hsa-miR-106a & 1.68 & hsa-miR-142-3p & -8.05 \\
\hline 12 & hsa-miR-17-5p & 1.58 & hsa-miR-150 & -8.01 \\
\hline 13 & hsa-miR-18b & 1.53 & hsa-miR-497 & -8.00 \\
\hline 14 & hsa-miR-19a & 1.45 & hsa-miR-214 & -7.66 \\
\hline 15 & hsa-miR-221 & 1.34 & hsa-miR-199a & -7.43 \\
\hline 16 & hsa-miR-93 & 1.30 & hsa-miR-146b & -7.30 \\
\hline 17 & hsa-miR-20a & 1.26 & hsa-miR-133b & -7.06 \\
\hline 18 & - & - & hsa-miR-127 & -6.99 \\
\hline 19 & - & - & hsa-miR-199a* & -6.59 \\
\hline 20 & - & - & hsa-miR-381 & -6.56 \\
\hline 21 & - & - & hsa-miR-195 & -6.54 \\
\hline 22 & - & - & hsa-miR-648 & -6.46 \\
\hline 23 & - & - & hsa-miR-223 & -6.41 \\
\hline 24 & - & - & hsa-miR-135a & -5.99 \\
\hline 25 & - & - & hsa-miR-146a & -5.98 \\
\hline 26 & - & - & hsa-miR-136 & -5.21 \\
\hline 27 & - & - & hsa-miR-126 & -5.11 \\
\hline 28 & - & - & hsa-miR-29c & -5.04 \\
\hline 29 & - & - & hsa-miR-572 & -5.03 \\
\hline
\end{tabular}

miRNA, microRNA.

Tissue samples. A total of 40 pairs of primary gastric cancer and matched adjacent non-tumorous tissues were obtained from surgically treated gastric cancer patients diagnosed at the Department of Surgery, Ruijin Hospital, Shanghai Jiao Tong University School of Medicine from 2006 to 2008. All gastric cancer cases were clinically and pathologically proven. Each specimen was given a diagnosis and scored for tumor size, location, differentiation and pTNM stage. Other clinical characteristics were obtained from clinical records with patient permission. Table II shows detailed information on the patients and the clinicopathological parameters of the tumors. A total of 6 normal gastric epithelium biopsy tissues were collected from subjects who were referred for gastroscopy. The study was approved by the Ruijin Hospital Ethics Committee and patients provided informed consent.

RNA preparation and microRNA microarray. RNA extraction of cells and tissue samples were performed with the mirVana miRNA Isolation kit (Ambion, Austin, TX, USA) according the manufacturer's instructions. Small RNA molecules (smaller than $200 \mathrm{nt}$ ) were separated and purified from long RNA molecules (longer than $200 \mathrm{nt}$ ) using this procedure. RNA molecules of $100 \mathrm{ng}$ underwent microRNA expression profiling following the microRNA microarray protocol (Agilent Technologies, Santa Clara, USA). For dephosphorylation and ligation, 17 units of calf intestine alkaline phosphatase (GE Healthcare, Amersham, UK) and 20 units of T4 RNA ligase (Ambion) were used. Each sample was hybridized on Human microRNA Microarray v.2 (Agilent Technologies) containing probes for 723 human microRNAs. Slides were scanned using an Agilent G2565BA scanner and images were processed using Feature Extraction software v.9.5.3.1 (Agilent Technologies).

Quantitative real-time PCR (Q-PCR) analysis of mature miRNAs. Total RNA, including miRNA, was extracted from cells and tissues using an miRNeasy kit (Qiagen, Germany). The miRNAs and other noncoding RNAs were polyadenylated by poly(A) polymerase and subsequently converted into cDNA by reverse transcriptase with oligo-dT priming (Qiagen). The 
cDNA was then used for real-time PCR quantification of any miRNA, noncoding RNA or mRNA. The cDNA served as the template for real-time PCR. Analysis was performed on an ABI Prism 7000 (Applied Biosystems, Foster City, CA, USA) using the miScript Primer Assay in combination with the miScript SYBR-Green PCR kit (Qiagen). miRNAs were amplified using the miScript Universal Primer, which primes from the universal tag sequence, with the miRNA-specific primer (Qiagen). Data were analyzed using the comparative $\mathrm{Ct}$ method. The specificity of the resulting PCR products was confirmed by melting curves.

Statistical and bioinformatics analysis. Data were analyzed by subtracting the background and normalizing the signals using a locally-weighted regression (LOWESS) filter. They were then $\log 2$-transformed and normalized for between-array comparison using quantile normalization. microRNAs with p-values $<0.05$ were selected for further analysis. We used ANOVA and class prediction statistical tools to identify significantly different levels of miRNA expression in gastric cancer cell lines and normal gastric mucosa. Microarray data were hierarchically clustered using the GeneCluster program, and dendrograms and expression maps were generated using Java Treeview version 1.0 (Department of Genetics, Stanford University School of Medicine, Stanford, CA, USA) (14).

To perform survival analysis and generate Kaplan-Meier survival curves, miRNA levels measured on Q-PCR were converted into discrete variables by splitting the samples into two classes (high and low expression) using the respective mean level of miRNA expression as the threshold. Survival curves were compared by log-rank analysis. A $95 \%$ confidence interval used in tests for significance.

\section{Results}

miRNA expression signature of gastric cancer cell lines relative to normal gastric mucosa. We performed an miRNA microarray to evaluate the miRNA expression profiles of gastric cancer cell lines and normal gastric mucosa. The miRNA expression pattern was found to be significantly different. The miRNA microarray identified 17 miRNAs that were upregulated in gastric cancer cell lines and 146 that were downregulated relative to normal gastric mucosa. Table I lists all 17 upregulated miRNAs and $29 \log$ ratio <-5.0 downregulated miRNAs.

Cluster analysis based on miRNAs differentially expressed between gastric cancer cell lines and normal gastric mucosa demonstrated a general difference between the 2 groups (Fig. 1). The expression patterns appeared to be consistent within the 9 gastric cancer cell lines and within the 6 normal gastric mucosa lines, and there was a more clear distinction between the 2 groups.

Expression of candidate miRNAs in gastric cancer cell lines and tissues. To validate the microarray findings, Q-PCR was undertaken for the 9 gastric cancer cell lines, 6 normal gastric mucosa, and a further 40 gastric cancer tissues and matched adjacent non-tumorous tissues for 41 candidate miRNAs. Four miRNAs had no commercial miRNA-specific primer: hsa-miR-17-5p, hsa-miR-301, hsa-miR-199a* and

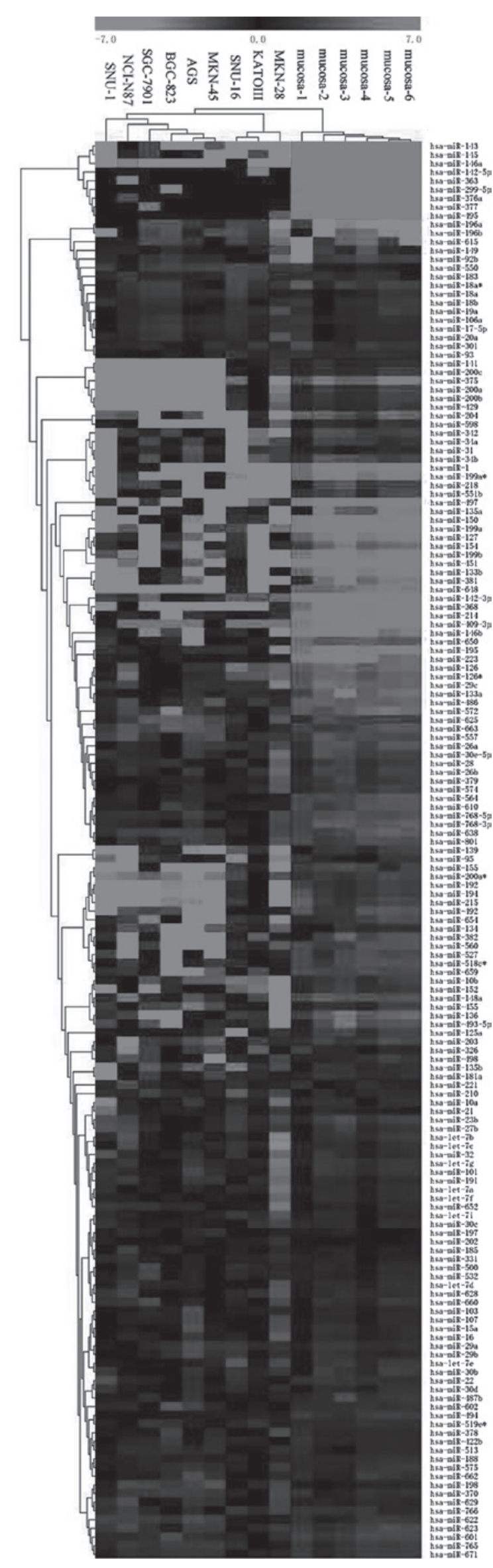

Figure 1. Alterations in miRNAs expression profiles across 9 gastric cancer cell lines and 6 normal gastric mucosa. Gastric cancer cell lines were NCIN87, SNU-1, SNU-16, AGS, KATOIII, MKN45, MKN28, BGC-823 and SGC-7901. 


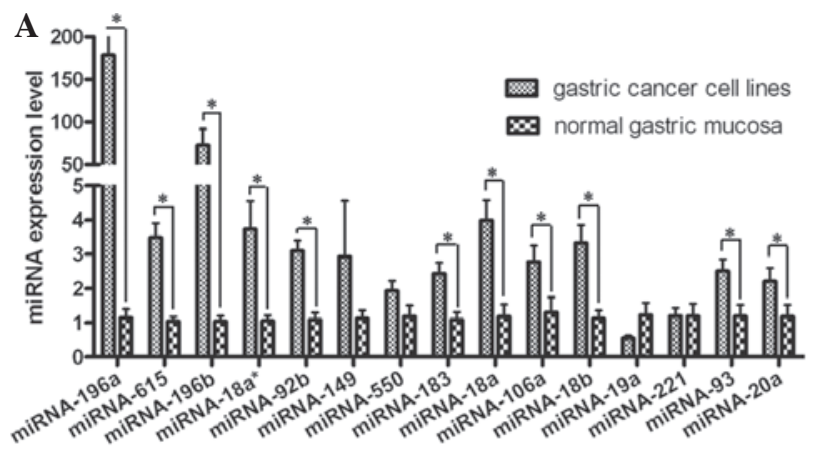

B
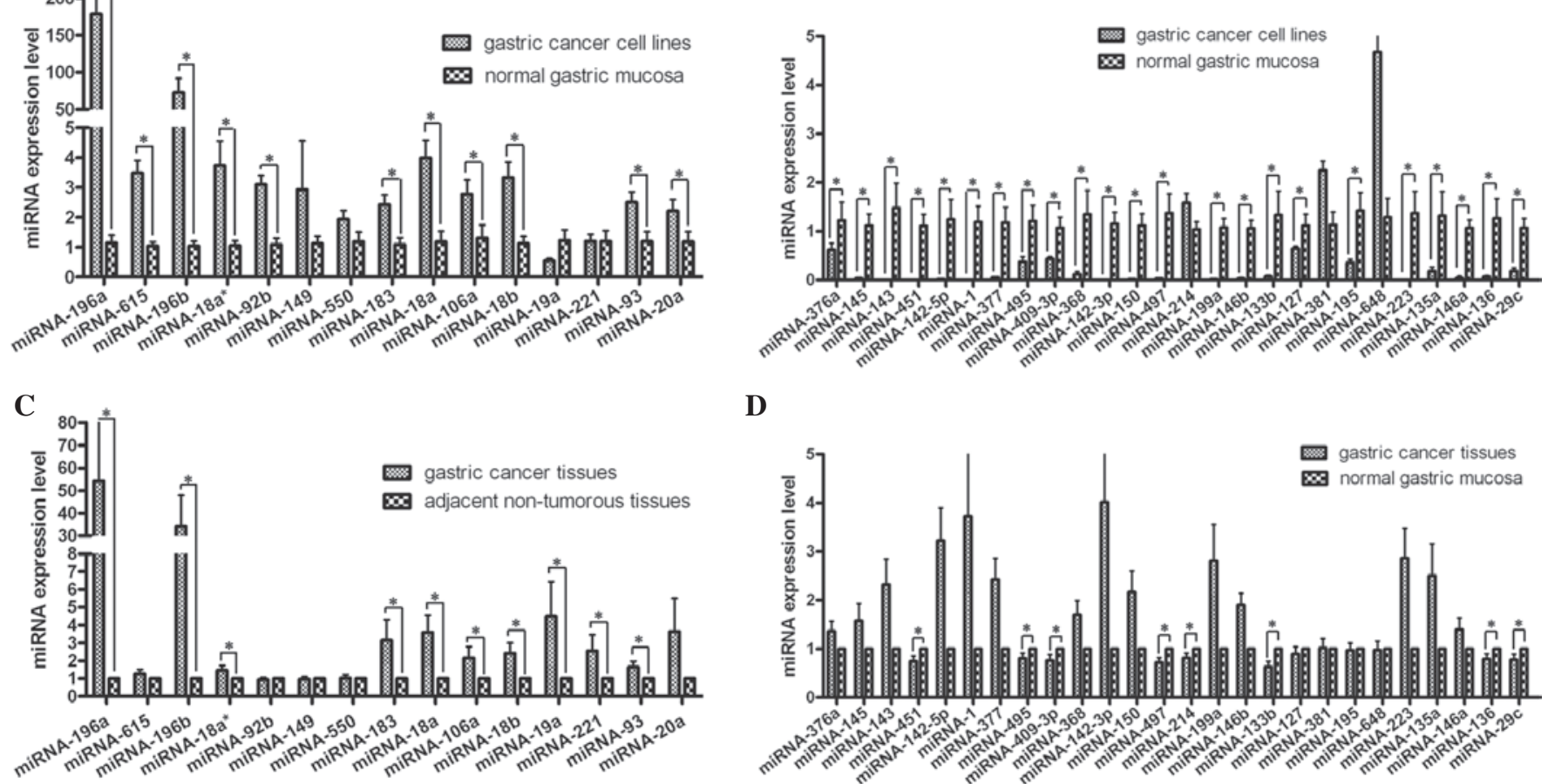

D

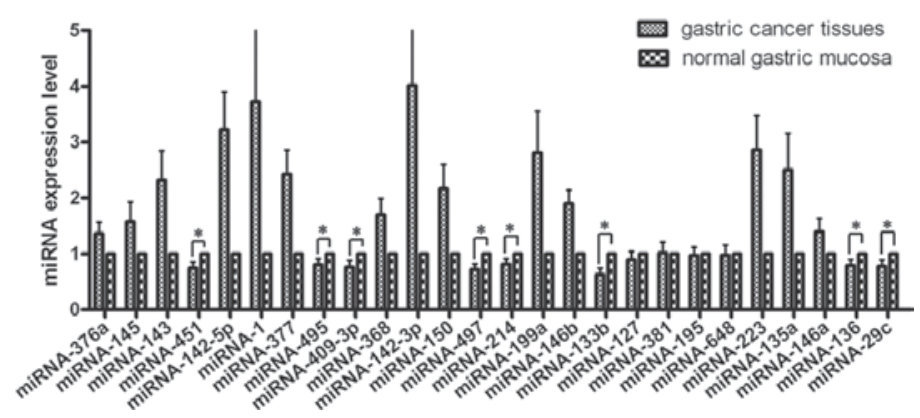

Figure 2. Expression of candidate miRNAs in gastric cancer cell lines and tissues. (A) Expression levels of 15 upregulated miRNAs in gastric cancer cell lines and normal gastric mucosa. (B) Expression levels of 26 downregulated miRNAs in gastric cancer cell lines and normal gastric mucosa. (C) Expression levels of 15 upregulated miRNAs in 40 gastric cancer tissue samples matched against adjacent non-tumorous tissues. (D) Expression levels of 26 downregulated miRNAs in 40 gastric cancer tissue samples matched against adjacent non-tumorous tissues. These results were repeated in three independent experiments. Error bars indicate the standard error around the mean. " $\mathrm{P}<0.05$, by paired Student's t-test.

hsa-miR-572. hsa-miR-126 is being studied by another research team at our facility. Q-PCR-measured expression of the majority of selected miRNAs in gastric cancer cell lines and normal gastric mucosa was consistent with microarray results. The incidence of upregulated miRNAs and downregulated miRNAs across the cancerous and normal samples was $73.33 \%(11 / 15)$ and $88.46 \%$ (23/26), respectively (Fig. 2A and B). Moreover, as expected, certain discrepancies were detected when miRNA microarray data were validated with Q-PCR-measured expression levels. Nevertheless, the expression level of 41 miRNAs using Q-PCR showed a great deal of diversity when compared to microarray data. The incidence of upregulated miRNAs was $66.67 \%(10 / 15)$ and the incidence of downregulated miRNAs was $30.77 \%(8 / 26)$ (Fig. 2C and D). This was likely to be due to the high heterogeneity of cancer tissues relative to cancer cell lines. According to the results of miRNA microarray and Q-PCR, 8 upregulated miRNAs (hsa-miR-196a, hsa-miR-196b, hsa-miR-18a*, hsa-miR-183, hsa-miR-18a, hsa-miR-106a, hsa-miR-18b and hsa-miR-93) and 7 downregulated miRNAs (hsa-miR-451, hsa-miR-495, hsa-miR-409-3p, hsa-miR-497, hsa-miR-133b, hsa-miR-136 and hsa-miR-29c) were identified.

Clinicopathological association of expression levels of 15 miRNAs in gastric cancer. The expression levels of 15 miRNAs were assessed by Q-PCR in 40 primary gastric cancer samples and their matched adjacent non-tumorous counterparts. The mean miRNA expression level and the correlation between miRNA expression and clinicopatho- logical features is shown in Table II. Among the upregulated miRNAs, miR-196a and miR-183 were expressed more highly in male than in female patients $(\mathrm{p}<0.05)$. miR-196b expression was correlated with gender, differentiation and degree of infiltration $(p=0.014, p<0.001$ and $p=0.001$, respectively). miR-18a* was more highly expressed in older patients ( $\geq 60$ years old) and miR-93 was correlated with differentiation and degree of infiltration $(\mathrm{p}<0.05)$. miR-18a and miR-18b were associated with age, differentiation and degree of infiltration $(\mathrm{p}<0.05)$. miR-106a had an association with age, gender, differentiation and degree of infiltration $(\mathrm{p}<0.05)$. Among the downregulated miRNAs, miR-451 was correlated with gender $(\mathrm{p}<0.001)$ and miR-495 was correlated with age $(\mathrm{p}=0.034)$. The miR-409-3p was associated with Borrmann type and miR-497 with the degree of infiltration and TNM stage $(\mathrm{p}<0.05)$. The miR-133b was expressed less in serosal-invaded samples than in subserosal samples $(\mathrm{p}=0.002)$.

Survival curve of selected miRNAs. To determine the impact of these 15 miRNAs on survival, Kaplan-Meier survival curves were created and analyzed. The curves were compared using log-rank analysis and the binomial variable of high or low expression relative to the mean expression of each miRNA based on the results of Q-PCR. Survival information was available for 36 out of 40 patients. One miRNA of interest was identified: hsa-miR-93 (Fig. 3). High expression of hsa-miR-93, which was observed in $70 \%(25 / 36)$ of the gastric cancer patients, was correlated with 16 months median survival time compared with 40 months for the low expres- 
Table II. Clinicopathological associations of the expression of 15 miRNAs using quantitative real-time PCR in gastric cancer.

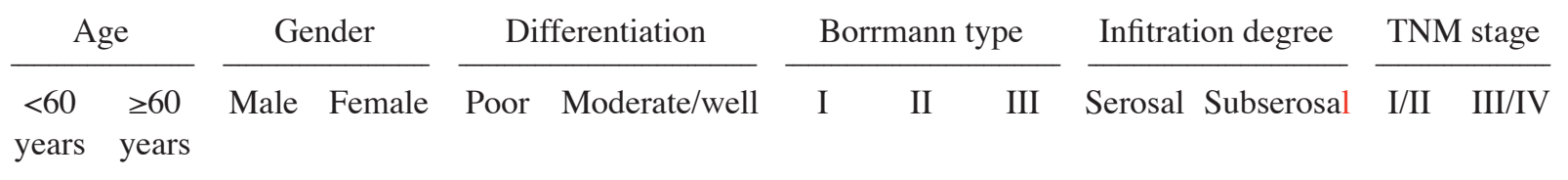

\begin{tabular}{|c|c|c|c|c|c|c|c|c|c|c|c|c|c|}
\hline \multicolumn{14}{|l|}{ miR-196a } \\
\hline Mean & 21.45 & 72.53 & 94.12 & 16.26 & 60.68 & 24.54 & 70.88 & 21.73 & 18.75 & 19.81 & 63.06 & 15.45 & 69.41 \\
\hline P-value & 0.211 & - & 0.037 & - & 0.385 & - & 0.390 & - & - & 0.346 & - & 0.193 & - \\
\hline \multicolumn{14}{|l|}{ miR-196b } \\
\hline Mean & 12.77 & 46.24 & 57.98 & 11.66 & 23.01 & 85.48 & 44.61 & 12.35 & 15.92 & 73.42 & 24.11 & 9.56 & 43.92 \\
\hline P-value & 0.093 & - & 0.014 & - & 0 & - & 0.188 & - & - & 0.001 & - & 0.099 & - \\
\hline \multicolumn{14}{|l|}{ miR-18a* } \\
\hline Mean & 1.01 & 1.73 & 1.76 & 1.20 & 1.51 & 1.32 & 1.69 & 0.94 & 1.28 & 1.19 & 1.55 & 1.10 & 1.62 \\
\hline P-value & 0.038 & - & 0.108 & - & 0.777 & - & 0.150 & - & - & 0.717 & - & 0.539 & - \\
\hline \multicolumn{14}{|l|}{ miR-183 } \\
\hline Mean & 3.35 & 3.04 & 4.73 & 1.65 & 2.62 & 5.59 & 3.67 & 2.50 & 1.21 & 4.81 & 2.72 & 2.22 & 3.51 \\
\hline P-value & 0.499 & - & 0.012 & - & 0.083 & - & 0.385 & - & - & 0.120 & - & 0.370 & - \\
\hline \multicolumn{14}{|l|}{ miR-18a } \\
\hline Mean & 1.86 & 4.58 & 4.01 & 3.21 & 2.61 & 8.15 & 3.52 & 4.14 & 2.94 & 6.62 & 2.82 & 3.60 & 3.60 \\
\hline P-value & 0.026 & - & 0.198 & - & 0 & - & 0.639 & - & - & 0 & - & 0.831 & - \\
\hline \multicolumn{14}{|l|}{ miR-106a } \\
\hline Mean & 1.02 & 2.80 & 2.81 & 1.54 & 1.65 & 4.48 & 2.53 & 1.34 & 1.57 & 3.82 & 1.73 & 1.66 & 2.36 \\
\hline P-value & 0.033 & - & 0.035 & - & 0.001 & - & 0.334 & - & - & 0.003 & - & 0.501 & - \\
\hline \multicolumn{14}{|l|}{ miR-18b } \\
\hline Mean & 1.44 & 2.98 & 2.81 & 2.06 & 2.03 & 4.22 & 2.46 & 2.55 & 1.95 & 3.49 & 2.15 & 2.23 & 2.50 \\
\hline P-value & 0.036 & - & 0.064 & - & 0.009 & - & 0.740 & - & - & 0.013 & - & 0.902 & - \\
\hline \multicolumn{14}{|l|}{ miR-93 } \\
\hline Mean & 1.04 & 1.99 & 2.02 & 1.29 & 1.38 & 2.85 & 1.75 & 1.41 & 1.47 & 2.21 & 1.50 & 0.91 & 1.93 \\
\hline P-value & 0.189 & - & 0.162 & - & 0.001 & - & 0.516 & - & - & 0.001 & - & 0.339 & - \\
\hline \multicolumn{14}{|l|}{ miR-451 } \\
\hline Mean & 0.72 & 0.75 & 0.50 & 0.97 & 0.67 & 1.08 & 0.68 & 0.78 & 1.04 & 0.64 & 0.77 & 0.59 & 0.80 \\
\hline P-value & 0.651 & - & 0 & - & 0.321 & - & 0.152 & - & - & 0.504 & - & 0.332 & - \\
\hline \multicolumn{14}{|l|}{ miR-495 } \\
\hline Mean & 0.75 & 0.83 & 0.87 & 0.74 & 0.85 & 0.57 & 0.73 & 1.10 & 0.61 & 0.81 & 0.80 & 0.62 & 0.87 \\
\hline P-value & 0.034 & - & 0.801 & - & 0.210 & - & 0.574 & - & - & 0.879 & - & 0.374 & - \\
\hline \multicolumn{14}{|c|}{$\operatorname{miR}-409-3 p$} \\
\hline Mean & 0.56 & 0.87 & 0.79 & 0.74 & 0.73 & 0.90 & 0.77 & 0.87 & 0.45 & 0.56 & 0.82 & 0.93 & 0.70 \\
\hline P-value & 0.052 & - & 0.859 & - & 0.433 & - & 0.021 & - & - & 0.093 & - & 0.191 & - \\
\hline \multicolumn{14}{|l|}{ miR-497 } \\
\hline Mean & 0.86 & 0.64 & 0.73 & 0.71 & 0.78 & 0.44 & 0.71 & 0.72 & 0.81 & 0.31 & 0.83 & 0.43 & 0.83 \\
\hline P-value & 0.705 & - & 0.260 & - & 0.188 & - & 0.421 & - & - & 0.002 & - & 0.024 & - \\
\hline \multicolumn{14}{|l|}{ miR-133b } \\
\hline Mean & 0.53 & 0.69 & 0.66 & 0.60 & 0.61 & 0.71 & 0.63 & 0.50 & 0.89 & 0.20 & 0.74 & 0.61 & 0.64 \\
\hline P-value & 0.321 & - & 0.667 & - & 0.654 & - & 0.850 & - & - & 0.002 & - & 0.993 & - \\
\hline \multicolumn{14}{|l|}{ miR-136 } \\
\hline Mean & 0.83 & 0.77 & 0.66 & 0.91 & 0.76 & 0.95 & 0.82 & 0.83 & 0.49 & 0.69 & 0.82 & 0.77 & 0.80 \\
\hline P-value & 0.894 & - & 0.950 & - & 0.551 & - & 0.103 & - & - & 0.961 & - & 0.611 & - \\
\hline \multicolumn{14}{|l|}{ miR-29c } \\
\hline Mean & 0.74 & 0.79 & 0.79 & 0.76 & 0.80 & 0.64 & 0.67 & 0.74 & 1.52 & 0.50 & 0.85 & 0.59 & 0.85 \\
\hline P-value & 0.673 & - & 0.117 & - & 0.295 & - & 0.323 & - & - & 0.327 & - & 0.315 & - \\
\hline
\end{tabular}

miRNA, microRNA. 


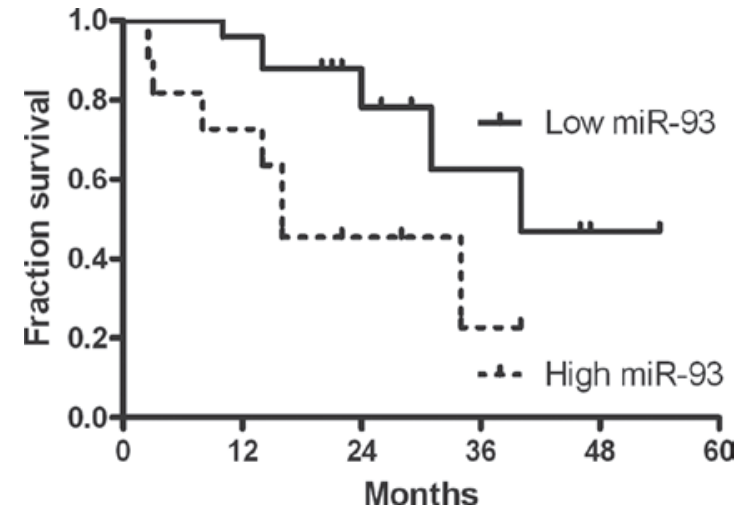

Figure 3. Kaplan-Meier overall survival curve for 36 patients with gastric cancer based on the expression of miRNAs. Survival curve at different expression levels of hsa-miR-93 (high and low); log-rank $\mathrm{p}=0.0214$. miRNA, microRNA.

sion group. The median survival time for all patients was 22.96 months.

\section{Discussion}

The role of miRNAs in various cancers has received extensive study in recent years. miRNAs play an important role in various physiological and developmental processes, such as cellular differentiation and organism development. They are capable of acting as either potent oncogenes or tumor suppressor genes (15-17). Guo et al reported that the mir-17-92 cluster, miR-20b, miR-106a, miR-21, miR-106b, miR-18b, miR-421, miR-340* and miR-658 were highly expressed in gastric cancer tissues (18). miR-106b, miR-93 and miR-25 overexpression caused gastric cancer cells to be less sensitive to TGF $\beta$-dependent cell-cycle arrest and apoptosis (19).

To understand the role of miRNAs in gastric cancer, a signature miRNA expression profile of gastric cancer cell lines was created and compared to the miRNA expression profile of normal gastric mucosa. Our team found that certain miRNAs were closely related to the development of gastric cancer. These results contribute greatly to understanding the molecular basis of human gastric cancer and suggest that aberrant expression of miRNA may be important in the pathogenesis of gastric cancer. Moreover, hsa-miR-93 was found to be significantly associated with duration of survival. This was determined from a quantitative examination of long- and short-term survivors.

Since one miRNA is capable of modulating the activity of multiple genes, miRNA profiling may provide new and complementary insights into the genetic pathways involved in cancer development (20). Aberrant miRNA expression patterns have been described in a variety of hematological and solid-organ malignancies. The present study contributes to the growing understanding of the role of miRNAs in oncogenesis and describes the global expression patterns of miRNAs in gastric cancer. More importantly, data such as ours may help guide clinicians when determining who should or should not receive aggressive therapy.

\section{Acknowledgements}

This study was supported by the National Natural Scientific Foundation of China (no. 30872476), the National High Technology Research and Development Program of China (863 Program; no. 2006AA02A301 and no. 2007AA02Z179), the Science and Technology Commission of Shanghai Municipality (no. 09DZ1950100, no. 09DZ2260200 and no. 10JC1411100) and the Shanghai Key Discipline (S30204) and Key Projects in the National Science and Technology Pillar Program (no. 2008BA152B03).

\section{References}

1. Tan YK and Fielding JW: Early diagnosis of early gastric cancer. Eur J Gastroenterol Hepatol 18: 821-829, 2006.

2. Pillai RS: MicroRNA function: multiple mechanisms for a tiny RNA? RNA 11: 1753-1761, 2005.

3. Filipowicz W, Bhattacharyya SN and Sonenberg N: Mechanisms of post-transcriptional regulation by microRNAs: are the answers in sight? Nat Rev Genet 9: 102-114, 2008.

4. Wijnhoven BP, Michael MZ and Watson DI: MicroRNAs and cancer. Br J Surg 94: 23-30, 2007.

5. Bushati $\mathrm{N}$ and Cohen SM: microRNA functions. Annu Rev Cell Dev Biol 23: 175-205, 2007.

6. Calin GA, Sevignani C, Dumitru CD, et al: Human microRNA genes are frequently located at fragile sites and genomic regions involved in cancers. Proc Natl Acad Sci USA 101: 2999-3004, 2004.

7. Calin GA, Liu CG, Sevignani C, et al: MicroRNA profiling reveals distinct signatures in $\mathrm{B}$ cell chronic lymphocytic leukemias. Proc Natl Acad Sci USA 101: 11755-11760, 2004.

8. Yanaihara N, Caplen N, Bowman E, et al: Unique microRNA molecular profiles in lung cancer diagnosis and prognosis. Cancer Cell 9: 189-198, 2006.

9. Fassan M, Baffa R, Palazzo JP, Lloyd J, Crosariol M, Liu CG, Volinia S, Alder H, Rugge M, Croce CM and Rosenberg A: MicroRNA expression profiling of male breast cancer. Breast Cancer Res 11: R58, 2009.

10. Michael MZ, O'Connor SM, van Holst Pellekaan NG, Young GP and James RJ: Reduced accumulation of specific microRNA in colorectal neoplasia. Mol Cancer Res 1: 882-891, 2003.

11. Zamore PD and Haley B: Ribo-genome: the big world of small RNAs. Science 309: 1519-1524, 2005.

12. Siomi $\mathrm{H}$ and Siomi MC: Posttranscriptional regulation of microRNA biogenesis in animals. Mol Cell 38: 323-332, 2010.

13. Lu J, Getz G, Miska EA, et al: MicroRNA expression profiles classify human cancers. Nature 435: 834-838, 2005.

14. Eisen MB, Spellman PT, Brown PO and Botstein D: Cluster analysis and display of genome-wide expression patterns. Proc Natl Acad Sci USA 95: 14863-14868, 1998.

15. Kloosterman WP and Plasterk RH: The diverse functions of microRNAs in animal development and disease. Dev Cell 11: 441-450, 2006.

16. Volinia S, Calin GA, Liu CG, et al: A microRNA expression signature of human solid tumors defines cancer gene targets. Proc Natl Acad Sci USA 103: 2257-2261, 2006.

17. Esquela-Kerscher A and Slack FJ: Oncomirs - microRNAs with a role in cancer. Nat Rev Cancer 6: 259-269, 2006.

18. Guo J, Miao Y, Xiao B, Huan R, Jiang Z, Meng D and Wang Y: Differential expression of microRNA species in human gastric cancer versus non-tumorous tissues. J Gastroenterol Hepatol 24: 652-657, 2009.

19. Petrocca F, Visone R, Onelli MR, et al: E2F1-regulated microRNAs impair TGF $\beta$-dependent cell-cycle arrest and apoptosis in gastric cancer. Cancer Cell 13: 272-286, 2008.

20. Michlewski G, Guil S, Semple CA and Cáceres JF: Posttranscriptional regulation of miRNAs harboring conserved terminal loops. Mol Cell 32: 383-393, 2008. 